\title{
Effect of COVID-19 Pandemic on Anxiety Levels of Children with Cystic Fibrosis and Healthy Children
}

\author{
Mürüvvet Cenk ${ }^{1}$, Cansu Ylmaz Yegit ${ }^{2}$, Almala Ergenekon ${ }^{1}$, Ayse Toksoy Aksoy $^{1}$, Gulcin \\ Bilicen $^{1}$, Yasemin Gokdemir ${ }^{2}$, Ela Erdem Eralp ${ }^{3}$, Ayse Rodopman Arman ${ }^{1}$, Fazilet \\ Karakoc $^{3}$, and Bulent Karadag ${ }^{3}$ \\ ${ }^{1}$ Marmara University School of Medicine \\ ${ }^{2}$ Marmara University \\ ${ }^{3}$ Marmara University Faculty of Medicine
}

October 8, 2020

\begin{abstract}
Background Cystic fibrosis $(\mathrm{CF})$ is a chronic disease causing recurrent respiratory tract infections. Viral respiratory tract infections are more severe in CF. The first case of COVID-19 was seen in Turkey on March 11, 2020 and nationwide school closure and lockdown were implemented. School closure and home confinement might have adverse effects on children's physical and mental health. In this study, we aimed to compare the effect of COVID-19 pandemic on psychological reactions of CF patients and healthy controls. Methods This is a controlled cross-sectional study including 7-18 year-old children with CF. The survey included questions regarding family environment and peer relations, self care and psychological reactions to COVID-19 pandemic. The questionnaire was applied to children via telephone call under parental supervision. Results We evaluated $132 \mathrm{CF}$ patients and 135 their healthy peers. Mean age was $11.5 \pm 2.9$ years in CF group and $11.8 \pm 3.2$ years in control group $(\mathrm{p}=0.98)$. There were 55 girls $(41.7 \%)$ in $\mathrm{CF}$ group and 81 girls $(60 \%)$ in control group $(\mathrm{p}=0.027)$. The socioeconomic status of families was similar. CF patients was feeling less anxious about the COVID-19 pandemic compared to controls ( $\mathrm{p}=0.01)$. CF patients were found to be less anxious for family members having the risk of COVID-19, less upset for the school closure, less anxious about the COVID-19 pandemic ( $\mathrm{p}<0.001,0.02,0.01$ respectively). Conclusion CF patients seem to show more resilience in coping with the pandemic. Appropriate psychological support should be provided to them and resilience strategies in coping with the pandemic should be nurtured.
\end{abstract}

\section{TITLE PAGE}

The Effect of COVID-19 Pandemic on Anxiety Levels of Children with Cystic Fibrosis and Healthy Children

Muruvvet Cenk ${ }^{1}$, Cansu Yilmaz Yegit ${ }^{1}$, Almala Pinar Ergenekon ${ }^{1}$, Ayse Toksoy Aksoy ${ }^{2}$, Gulcin Bilicen ${ }^{3}$, Yasemin Gokdemir ${ }^{1}$, Ela Erdem Eralp ${ }^{1}$, Ayse Rodopman Arman ${ }^{2}$, Fazilet Karakoc ${ }^{1}$, Bulent Karadag ${ }^{1}$

${ }^{1}$ Division of Pediatric Pulmonology; Marmara University, School of Medicine, Istanbul, Turkey

${ }^{2}$ Department of Child and Adolescent Psychiatry, Marmara University, School of Medicine, Istanbul, Turkey

${ }^{3}$ Marmara University, School of Medicine, Istanbul, Turkey

Correspondence to: Muruvvet Cenk, MD

Kordonboyu mah Ankara cad No:147/196 Kartal/Istanbul

TURKIYE 
Tel (mobile): +90 5364191641

Email: muruvvetcenk@gmail.com

\section{Abstract \\ Background}

Cystic fibrosis $(\mathrm{CF})$ is a chronic disease causing recurrent respiratory tract infections. Viral respiratory tract infections are more severe in CF. The first case of COVID-19 was seen in Turkey on March 11, 2020 and nationwide school closure and lockdown were implemented. School closure and home confinement might have adverse effects on children's physical and mental health. In this study, we aimed to compare the effect of COVID-19 pandemic on psychological reactions of CF patients and healthy controls.

\section{Methods}

This is a controlled cross-sectional study including 7-18 year-old children with CF. The survey included questions regarding family environment and peer relations, self care and psychological reactions to COVID19 pandemic. The questionnaire was applied to children via telephone call under parental supervision.

\section{Results}

We evaluated $132 \mathrm{CF}$ patients and 135 their healthy peers. Mean age was $11.5 \pm 2.9$ years in $\mathrm{CF}$ group and $11.8 \pm 3.2$ years in control group $(\mathrm{p}=0.98)$. There were 55 girls $(41.7 \%)$ in $\mathrm{CF}$ group and 81 girls $(60 \%)$ in control group $(\mathrm{p}=0.027)$. The socioeconomic status of families was similar. CF patients was feeling less anxious about the COVID-19 pandemic compared to controls $(\mathrm{p}=0.01)$. CF patients were found to be less anxious for family members having the risk of COVID-19, less upset for the school closure, less anxious about the COVID-19 pandemic ( $\mathrm{p}<0.001,0.02,0.01$ respectively).

\section{Conclusion}

CF patients seem to show more resilience in coping with the pandemic. Appropriate psychological support should be provided to them and resilience strategies in coping with the pandemic should be nurtured.

Key Words: Cystic Fibrosis, COVID-19, Anxiety, Resilience, Coping

\section{Introduction}

Cystic fibrosis (CF) is a chronic, life-shortening disease causing recurrent respiratory tract infections and loss of lung functions [1]. Previous meta-analyses and systematic reviews have shown that adults and children with chronic diseases have higher risk of depression and anxiety compared to community [2,3]. A number of studies reported increased anxiety and depression in CF pateints compared to healthy controls [4-6]. Facing a chronic disease such as $\mathrm{CF}$ on a daily basis cause significant emotional burden which can be attributed to large number of treatments, hospitalizations, multiple diagnostic procedures, chronic respiratory symptoms, a lower body mass index [6].

Viral respiratory tract infections are more severe in patients with CF compared to general population. Influenza pandemic in 2009, caused significant morbidity in patients with CF and in a subgroup of patients with severe lung disease, H1N1 infection was associated with respiratory deterioration, intensive care admission and even death [7,8]. Coronavirus disease-19 (COVID-19) has emerged in China and been spreading all over the world since December 2019. The first case of COVID-19 infection has been confirmed in Turkey as of March 11, 2020. Although in most of the pediatric cases $(>90 \%)$ the disease is asymptomatic or presented with signs of upper respiratory tract infection symptoms, in a small number of children (\%0.6), the infection may progress into critical disease causing respiratory insufficiency and death [9].

During COVID-19 pandemic people are likely to experience fear of falling sick, dying or feelings of helplessness and a number of studies revealed a wide range of psychosocial impacts on people [4]. In a study by Wang et al, included 1210 respondents, psychological impact of the outbreak was present in 53.8\%. Moderate to severe depression, anxiety symptoms and stress levels were present in $16.5 \%, 28.8 \%$ and $8.1 \%$ of the participants 
respectively [10]. Another cross-sectional study in 7143 college students demonstrated that $21.3 \%$ had mild and $3.6 \%$ had moderate-to-severe anxiety symptoms where economic effects on daily life and delays in academic activities were positively associated with anxiety symptoms [11].

Although children seem to be less vulnerable than adults to COVID-19, initial reports from China indicate that children and adolescents also have been impacted psychologically, manifesting behavioral problem. Previous studies suggested that when children are out of school (eg, weekends and summer holidays), they are physically less active, have longer screen time, irregular sleep patterns, and less favourable feeding habits, resulting in weight gain and a loss of cardiorespiratory fitness [12,13].

Shuang-Jiang Zhou et al conducted a cross-sectional study including 8079 students aged 12-18 years during the COVID-19 pandemic period. The prevalence of depressive symptoms, anxiety symptoms, and a combination of depressive and anxiety symptoms was found $43.7 \%, 37.4 \%$, and $31.3 \%$, respectively [14]. Home confinement during pandemic appears to be another factor in increasing stress levels in children. In 2013, Sprang et al has been found that the post-traumatic stress scores of quarantined children was four times higher than those who were not quarantined [15].

Although the mortality of COVID-19 infection was found higher in patients with underlying diseases, until now, there is no evidence suggesting that the course of COVID-19 is more severe in CF patients compared to general population [16]. Clinical features of COVID-19 including dry cough, myalgia, and fever are quite distinct from the symptoms of $\mathrm{CF}$ and recognisable. However it is possible that mild disease might be labelled as within the normal spectrum of symptoms for that individual. However, COVID-19 infection still might cause more stress and anxiety in CF patients. To our knowledge there is only one study evaluating anxiety of CF patients during COVID-19 pandemic. Senkalfa et al compared the anxiety of 45 CF patients with 90 healthy control group and revealed that healthy children, aged 13-18 years had higher state anxiety scores than age-matched children with CF [17].

In this study, our aim was to evaluate the effect of COVID-19 pandemic on family environment and relations, self-care practises and peer relations, psychological reactions including anxiety and coping of CF patients and compare with healthy controls.

\section{Methods}

This is a controlled, cross-sectional, single center study including 7-18 year-old CF children who have been followed up in Selim Çöremen Cystic Fibrosis Center in Marmara University Faculty of Medicine, Division of Pediatric Pulmonology and their healthy peers.

Separate behavioral questionnaires for two different age groups (7-12 and 13-18 years), based on the child psychiatry mental health examination references [18], were formed by Marmara University Faculty of Medicine Child and Adolescent Psychiatry Department.

The questionnaire included questions regarding family environment and relations, self-care practises and peer relations during lockdown, and psychological reactions regarding COVID-19 pandemic. Data regarding presence of a health worker in the family, or a family member diagnosed with COVID-19, current anxiety level, sleep disturbances, change in appetite, activities in order to mitigate their anxiety (any attempt to have a new hobby etc.) and seek for a psychological help were also collected.

In $\mathrm{CF}$ patients, data regarding presence of pancreatic insufficiency, colonisation of Pseudomonas Aeruginosa and level of forced expiratory volume in second $1\left(\mathrm{FEV}_{1}\right)$ were collected from patients' medical records. Lung disease was categorised as mild, moderate and severe according to their baseline $\mathrm{FEV}_{1}$ measurements $\left(\mathrm{FEV}_{1}\right.$ 70-89 mild lung disease, $\mathrm{FEV}_{1} 40$ to $69 \%$ moderate lung disease, $\mathrm{FEV}_{1}<40 \%$ severe lung disease [19].

The data was collected between 30th April, 2020 and 15 th of May, in Turkey this time period was the peak of pandemic, schools were closed and education was online. We conducted phone survey by asking questions directly to the children under parental supervision. Children, younger than 7 years of age or who refuse to answer the questions or children with mental problems were excluded from the study. Informed consent 
was obtained from all parents following their agreement to participate the phone survey and the study was approved by the medical ethics committee of Marmara University (Protocol number 09.2020.549).

Data were analyzed by using the SPSS Statistical Package for Windows version 23.0. Numerical variables were expressed as mean \pm standart deviation or median. Caterogical variables were compared using the Chisquare test and expressed as numbers and percentages. A p value of less than 0.05 was considered statistically significant.

\section{Results}

This study included $132 \mathrm{CF}$ patients and 135 healthy peers. The mean age was $11.53 \pm 2.86$ and $11.76 \pm 3.15$ years respectively, in CF group and control group $(\mathrm{p}=0.98)$. There were 55 girls $(41.7 \%)$ in $\mathrm{CF}$ group and 81 girls $(60 \%)$ in control group $(\mathrm{p}=0.027)$.

Of the 132 CF patients, 95 (72\%) had pancreatic insuffiency, 41 (31.1\%) were colonised with Pseudomonas Aeruginosa, 59 (44.7\%) had normal $\mathrm{FEV}_{1}$ value, 40 (30.3\%) had mild, 23 (17.4\%) had moderate and $5(3.8 \%)$ of them had severe lung disease. There was no statistically significant difference in terms of severity of the disease and anxiety levels of CF patients $(\mathrm{p}>0.05)$. Comparison of psychosocial and psychological findings between CF patients and control group was presented in Table-1. CF patients were found to be less anxious for family members having the risk of COVID-19, less upset for the school closure, less anxious about the COVID-19 pandemic $(\mathrm{p}<0.001,0.02,0.01$ respectively). Control group was more overthinking about the pandemic, and feeling sad, lonely or reluctant to have playful time $(\mathrm{p}=0.03$ and 0.02 , respectively). Control group also tried to have new hobbies more compared to CF group $(\mathrm{p}=0.01)$.

In CF group $71(53.8 \%)$ of patients were children and $61(46.2 \%)$ were adolescents. Children were feeling more upset for the school closure $(\mathrm{p}=0.01)$ and more anxiety about the COVID-19 pandemic $(\mathrm{p}=0.008)$, had more appetite changes $(\mathrm{p}=0.03)$, expressed more reluctance of doing homework for online education $(\mathrm{p}=0.03)$, engaging activities to decrease their anxiety $(\mathrm{p}=0.01)$, tried to find new hobbies during lockdown $(\mathrm{p}=0.005)$ and needed psychological help $(\mathrm{p}=0.059)$ more than adolescents. Adolescents communicated with their friends via social media more than children $(\mathrm{p}=0.005)$. Comparison of psychological and psychosocial findings in $\mathrm{CF}$ group as children and adolescent was presented in Table-2.

In control group, $79(58.5 \%)$ of them were children and $56(41.5 \%)$ of them were adolescents. Although adolescents communicated with their friends from social media more than children $(\mathrm{p}=0.003)$, children felt happier for communicating online with their friends $(\mathrm{p}=0.02)$. Also children engaged new activities and needed for psychological help more than adolescents ( $\mathrm{p}$ value 0.008 and 0.11 respectively). Comparison of psychosocial and psychological findings in control group as children and adolescent was presented in Table-3

In our country examinations for high school and university admission are big stressors for adolescents. Twenty-four (39.4\%) of CF and 17 (30.4\%) of control group had admission examinations for high school or university. We also asked these adolescents if the pandemic, school closure and online education increased their anxiety. Although it was not statistically significant $(\mathrm{p}=0.21)$, exam anxiety was higher in control group compared to CF patients (46.4\% vs $24.6 \%)$.

\section{Discussion}

The COVID-19 has spread all over the world since the first quarter of 2020 and the uncertainty about the personal and global effects of COVID-19 in addition to psychological effect of quarantine caused fear and anxiety. Previous studies have suggested that the outbreaks can have many psychological effects like anxiety and depression [20,21]. Although a number of studies evaluated psychological impact of COVID-19 pandemic in children and adults, data regarding the effect of pandemic in patients with chronic disease is scarce [17].

In this study we evaluated the psychological reactions, family and peer relations, self-care practises and coping methods of CF patients during pandemic and compared with healthy controls. Symptoms of anxiety were unexpectedly higher in controls compared to CF patients. Almost $50 \%$ of the children in control group and $33 \%$ of the CF patients were feeling anxious about the COVID-19 pandemic. Feeling upset for school 
closure, worries for a family member having the risk of COVID-19 infection, feeling sad, lonely or reluctant to have playful time, overthinking about pandemic are all more common psychological reactions regarding pandemic in control group compared to CF patients.

Consistent to the results of current study, another study from Turkey, Senkalfa et al also reported lower anxiety scores regarding COVID-19 pandemic in CF patients compared to age-matched healthy control group [17]. Surprisingly lower anxiety scores in CF patients in both study may be related with a number of factors.

Since the viral respiratory tract infections tend to be more severe in CF patients with an increased risk of complications and a negative impact on lung function, in the first days of pandemic, our patients and their families were more concerned about the possible effect of COVID-19 infection. However until now, only few cases of COVID-19 infection in people with CF were reported, which may probably related with the efforts of families and patients to minimise social contacts [22]. It should also worthwhile to emphasize that reported cases are mainly adult CF patients and COVID-19 infection does not have an apparent effect on CF disease severity. It is not possible to identify protective factors and we need more data in order to determine the impact of COVID-19 on patients with CF. In cooperation with national registries, European Cystic Fibrosis Society (ECFS) Patient Registry has established an international dataset to identify factors that predict severity of COVID-19. However we assume that low numbers and mild course of COVID-19 infection caused a relieve in CF patients, which may partly explain lower anxiety levels in our patients.

During pandemic, consistent with international guidelines we recommended self-isolation for our CF patients. Recommendations for preventive measures such as using face masks and practising adequate hand hygiene were reinforced. The CF team cancelled routine clinic appointments to prevent unnecessary hospital visits and viral spread. Phone calls and email contacts were used to monitor the clinical condition of patients immediately after the lockdown. In a study from China, presence of psychological problems were negatively associated with the level of awareness of COVID-19 [14]. Wang et al revealed that wearing a mask and practicing hand hygiene decreases the level of anxiety in children older than 12 years old [10]. CF patients are used to follow instructions for infection control guidelines' including wearing masks and avoiding close inperson contact with other CF patients. The awareness regarding respiratory tract infections and familiarity to infection control measurements might be factors which decrease the level of anxiety in CF patients.

Psychosocial maladjustment might be another reason for lower anxiety levels in CF patients. Children with chronic physical disorders have twice the risk of psychosocial maladjustment compared with healthy children [23]. This maladjustment phenomenon might be an explanation for CF patients to give fewer psychological reactions in our study group in the case of pandemic, as they are already having longer times at home, mostly detached from the school or peer relations.

CF patients always facing with a serious health problem and associated anxiety symptoms, hovewer, healthy peers has been encountered fear of disease for the first time in their life during pandemic which can also partly explain relatively lower levels of anxiety in CF patients compared to healthy controls.

Although no significant difference was found between $\mathrm{CF}$ and control groups regarding family environment and relations or self care and peer relations, it is important to emphasize that almost half of the children were having apetite changes in both groups and incidence of sleeping problem were $10 \%$ in CF patients and $15 \%$ in control group. In our country, during disease outbreak, right after the school closure and home confinement, home schooling plan has been implemented. In addition to academic learning, age appropriate and attractive videos may motivate children to have a healthy lifestyle at home by increasing physical activities, having a balanced diet, regular sleep pattern, and good personal hygiene.

Althoug CF patients had a lower tendency to need psychological help compared to control group, children in CF group had more anxiety symptoms compared to adolescents and required more psychological help than adolescents in both groups. During pandemic children are constantly exposed to pandemic-related news, so having direct conversations with children about these issues could alleviate their anxiety. Home confinement, with the right parenting approaches could be a good opportunity to enhance family interactions and improve 
their self-sufficiency skills of children [24].

Resilience is the ability of properly adapt and react positively to adversity, trauma, individual or society tragedies, including serious health threats [25-26]. Coping responses mean the behaviours, thoughts and feelings that individuals adopt to avoid being harmed by life stressors [27]. In this study, we found that CF patients seem more resilient and developed adaptive coping strategies with the pandemic. Mental health of children and adolescents constitute the basis for future adult mental health and related to quality of life, productivity, and well-being as well as compliance to treatment in the presence of a chronic health condition. Therefore, it is a vital effort to understand the psychological reactions of CF patients during the unusual period of pandemic, in addition to the chronic illness status.

Current study has a few limitations. First, although the CF and control groups are aged-matched, they are not gender-matched. It has been shown that girls experience more anxiety than boys in previous studies [28-29]. Hovewer, since the anxiety levels of girls in our study were similar in CF and control groups, we assumed that gender difference has not been an important impact on our results.

Second, this was a phone survey and participants were recruited under parental supervision. Since it was a self-report assessment, the indicated levels of anxiety may not always be consistent with the assessment of mental health by a professional.

Several studies reported increased anxiety and depression in CF pateints compared to healthy controls and routine screening is strogly recommended, [4-6]. In a recent unpublished study, before pandemic, we revealed that moderate to severe depression and moderate anxiety was peresent in $25.5 \%$ and $17.6 \%$ of 50 adolescent CF patients respectively in our center [30].

Current study revealed that CF patients had lower anxiety symptoms and they were more resilient in coping during pandemic than their healthy peers.

In conclusion, although acute events such as pandemics may not seem to increase baseline anxiety and not associated with an important physcological impact in CF patients compared to heathy population, lack of psychological support in routine care may lead to higher levels of anxiety and depression in long term.

\section{Conflict of Interest}

There is no conflict of interest.

\section{References}

1. Flume PA, Mogayzel PJ Jr, Robinson KA, Goss CH, Rosenblatt RL, Kuhn RJ, Marshall BC, Clinical Practice Guidelines for Pulmonary Therapies Committee. Cystic fibrosis pulmonary guidelines: treatment of pulmonary exacerbations. Am J Respir Crit Care Med. 2009 Nov 1;180(9):802-8. Doi: 10.1164/rccm.200812-1845PP. Epub 2009 Sep 3.

2. Pinquart M, Shen Y. Depressive symptoms in children and adolescents with chronic physical illness: an updated meta-analysis. J Pediatr Psychol 2011;36:375-84. doi:10.1093/jpepsy/jsq104.

3. Moussavi S, Chatterji S, Verdes E, et al. Depression, chronic diseases, and decrements in health: results from the World Health Surveys. Lancet 2007;370:851-8. doi:10.1016/S0140-6736(07)61415-9.

4. Şenses-Dinç G, Özçelik U, Çak T, et al. Psychiatric morbidity and quality of life in children and adolescents with cystic fibrosis. Turk J Pediatr . 2018;60(1):32-40. doi:10.24953/turkjped.2018.01.005.

5. Besier T, Goldbeck L. Anxiety and depression in adolescents with CF and their caregivers. $J$ Cyst Fibros . 2011;10(6):435-442. doi:10.1016/j.jcf.2011.06.012.

6. Catastini P, Di Marco S, Furriolo M, et al. The prevalence of anxiety and depression in Italian patients with cystic fibrosis and their caregivers. Pediatr Pulmonol . 2016;51(12):1311-1319. doi:10.1002/ppul.23566.

7. Colombo C, Battezzati PM, Lucidi V, et al. Influenza A/H1N1 in patients with cystic fibrosis in Italy: a multicentre cohort study. Thorax . 2011;66(3):260-261. doi:10.1136/thx.2010.157032. 
8. Bucher J, Boelle PY, Hubert D, et al. Lessons from a French collaborative case-control study in cystic fibrosis patients during the 2009 A/H1N1 influenza pandemy. BMC Infect Dis . 2016;16:55. Published 2016 Feb 1. doi:10.1186/s12879-016-1352-2.

9. Dong Y, Mo X, Hu Y, et al. Epidemiology of COVID-19 Among Children in China. Pediatrics . 2020;145(6):e20200702. doi:10.1542/peds.2020-0702.

10. Wang C, Pan R, Wan X, et al. Immediate Psychological Responses and Associated Factors during the Initial Stage of the 2019 Coronavirus Disease (COVID-19) Epidemic among the General Population in China. Int J Environ Res Public Health. 2020;17(5):1729. doi:10.3390/ijerph17051729.

11. Cao W, Fang Z, Hou G, et al. The psychological impact of the COVID-19epidemic on college students in China. Psychiatri Res . 2020 May;287:112934. doi:10.1016/j.psychres.2020.112934. Epub 2020 Mar 20 .

12. Brazendale K, Beets MW, Weaver RG, et al. Understanding differences between summer vs. school obesogenic behaviors of children: the structured days hypothesis. Int $J$ Behav Nutr Phys Act . 2017;14(1):100. Published 2017 Jul 26. doi:10.1186/s12966-017-0555-2.

13. Wang G, Zhang J, Lam SP, et al. Ten-Year Secular Trends in Sleep/Wake Patterns in Shanghai and Hong Kong School-Aged Children: A Tale of Two Cities. J Clin Sleep Med . 2019;15(10):1495-1502. doi:10.5664/jcsm.7984.

14. Zhou SJ, Zhang LG, Wang LL, et al. Prevalence and socio-demographic correlates of psychological health problems in Chinese adolescents during the outbreak of COVID-19. Eur Child Adolesc Psychiatry . 2020;29(6):749-758. doi:10.1007/s00787-020-01541-4.

15. Sprang G, Silman M. Posttraumatic stres disorder in parents and youth after health related disasters. Disaster Med Public Health Prep2013;7(1): 105-10. doi:10.1017/dmp.2013.22.

16. Colombo C, Burgel PR, Gartner S, et al. Impact of COVID-19 on people with cystic fibrosis. The Lancet Respir Med. 2020 April;S2213-2600(20)30177-6. doi:10.1016/S2213-2600(20)301776.

17. Senkalfa BP,Eyuboglu TB, Aslan AT, et al. Effect of the COVID-19 Pandemic on anxiety among children with cystic fibrosis and their mothers. Pediatr Pulmonol. 2020;55(8):2128-34. Doi:10.1002/ppul.24900.

18. T. Lempp, D. de Lange, D. Radeloff, C. Bachmann. The clinical examination of children, adolescents and their families J.M. Rey (Ed.), IACAPAP e-Textbook of child and adolescent mental health, International Association for Child and Adolescent Psychiatry and Allied Professions, Geneva (2012).

19. Cystic Fibrosis Foundation Patient Registry. 2018 Annual Data Report, 2018.

20. Rubin GJ, Amlôt R, Page L, et al. Public perceptions, anxiety, and behaviour change in relation to the swine flu outbreak: cross sectional telephone survey. BMJ 2009; 339:b2651. doi:https://doi.org/10.1136/bmj.b2651.

21. Wheaton MG, Abramowitz JS, Berman NC, et al. Psychological Predictors of Anxiety in Response to the H1N1 (Swine Flu) Pandemic. Cogn Ther Res 36, 210-218 (2012).https://doi.org/10.1007/s10608011-9353-3.

22. European Cystic Fibrosis Society. COVID-CF Project in Europe.https://www.ecfs.eu/covid-cf-projecteurope.

23. Pless IB, Nolan T. Revision, replication and neglect-research on maladjustment in chronic illness. $J$ Child Psychol Psychiatry . 1991;32(2):347-365. doi:10.1111/j.1469-7610.1991.tb00312.x.

24. Wang G, Zhang Y, Zhao J, et al. Mitigate the effects of home confinement on children during the COVID-19outbreak. Lancet . 2020;395 (10228):945-947.

25. Stewart M, Reid G, Mangham C. Fostering children's resilience. J Pediatr Nurs . 1997;12(1):21-31. doi:10.1016/S0882-5963(97)80018-8.

26. Traub F, Boynton-Jarrett R. Modifiable Resilience Factors to Childhood Adversity for Clinical Pediatric Practice. Pediatrics . 2017;139(5):e20162569. doi:10.1542/peds.2016-2569.

27. Abbott J, Dodd M, Gee L, Webb K. Ways of coping with cystic fibrosis: implications for treatment adherence. Disabil Rehabil . 2001;23(8):315-324. doi:10.1080/09638280010004171.

28. Bender PK, Reinholdt-Dunne, Esbjorn BH, et al. Emotion Dysregulation and Anxiety In Children and Adolescents: Gender Differences.Personality and Individual Differences . 53(3):284-88. 
Doi:10.1016/j.paid.2012.03.027.

29. Zahn-Waxler C, Shirtcliff EA, Marceau K. Disorders of childhood and adolescence: gender and psychopathology. Annu Rev Clin Psychol . 2008;4:275-303. doi:10.1146/annurev.clinpsy.3.022806.091358.

30. Ergenekon P, Yegit CY, Mursaloglu H, et al. "Kistik Fibrozisli Hastalar ve Ailelerinde Depresyon ve Anksiyete Bozukluklarmın Taranması" abstract presented at National Pediatric Respiratory Diseases and Cystic Fibrosis Annual Congress 2020. https://cocuksolunum2020.org/gorseller/files/KistikFibrozis-Bildiri-Kitabi.pdf.

\section{Hosted file}

COVID-19 Anxiety Tables.pdf available at https://authorea.com/users/365219/articles/485402effect-of-covid-19-pandemic-on-anxiety-levels-of-children-with-cystic-fibrosis-andhealthy-children 